To be published in:

Thermoelectric Materials--New Directions and Approaches

Edited by T.M. Tritt, G. Mahan, H.B. Lyon, Jr., and M.G. Kanatzidis

Materials Research Society Symposium Proceedings, 1997

\title{
Filled Skutterudite Antimonides: Validation of the Electron-Crystal Phonon-Glass Approach to New Thermoelectric Materials
}

D. Mandrus ${ }^{1}$, B.C. Sales ${ }^{1}$, V. Keppens ${ }^{1}$, B.C. Chakoumakos ${ }^{1}$, P. Dai ${ }^{1}$, L.A. Boatner ${ }^{1}$, R.K. Williams ${ }^{2}$, J.R. Thompson ${ }^{1}$, T.W. Darling ${ }^{3}$, A. Migliori ${ }^{3}$, M.B. Maple ${ }^{4}$, D.A. Gajewski ${ }^{4}$, and E.J. Freeman ${ }^{4}$

'Solid State Division, Oak Ridge National Laboratory, Oak Ridge, Tennessee 37831-6056

${ }^{2}$ Metals and Ceramics Division, Oak Ridge National Laboratory, Oak Ridge, Tennessee 37831-6056

${ }^{3}$ Materials Science and Technology Division, Los Alamos National Laboratory, Los Alamos, New Mexico 87454

${ }^{4}$ Department of Physics and Institute for Pure and Applied Physical Sciences, University of California, San Diego, La Jolla, California 92093

April 1997

"The submitted manuscript has been authored by a contractor of the
U.S. Government under contract DE-ACO5-960R22464. Accordingly.
the U.S. Government retains a nopexclusive, royalty-free license to
publish or reproduce the published form of this contribution, or sllow
others to do so, for U.S. Government purposes."

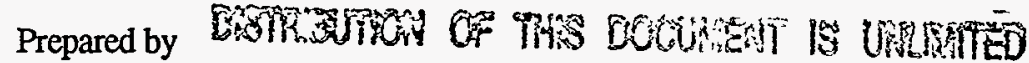

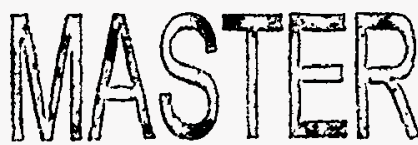

Solid State Division

Oak Ridge National Laboratory

P.O. Box 2008

Oak Ridge, Tennessee 37831-6056

managed by

LOCKHEED MARTIN ENERGY RESEARCH CORP.

for the

U.S. DEPARTMENT OF ENERGY

under contract DE-AC05-96OR22464

This research was sponsored by the Oak Ridge National Laboratory, managed by Lockheed Martin Energy Research Corp., for the U.S. Department of Energy, under contract DE-AC05-96OR22464. 


\section{DISCLAIMER}

Portions of this document may be illegible in electronic image products. Images are produced from the best available original document. 


\section{FILLED SKUTTERUDITE ANTIMONIDES: VALIDATION OF THE ELECTRON-CRYSTAL PHONON-GLASS APPROACH TO NEW THERMOELECTRIC MATERIALS}

D. MANDRUS*, B. C. SALES*, V. KEPPENS*, B. C. CHAKOUMAKOS*, P. DAI*, L. A. BOATNER*, R. K. WILLIAMS**, J. R. THOMPSON*, T. W. DARLING***, A. MIGLIORI***, M. B. MAPLE****, D. A. GAJEWSKI****, AND E. J. FREEMAN**** *Solid State Division, Oak Ridge National Laboratory, Oak Ridge, TN 37831

**Metals and Ceramics Division, Oak Ridge National Laboratory, Oak Ridge, TN 37831

***Materials Science and Technology Division, Los Alamos National laboratory, Los Alamos, NM 87545

****Department of Physics and Institute for Pure and Applied Physical Sciences, University of California, San Diego, La Jolla, CA 92093

\section{ABSTRACT}

After a brief review of the transport and thermoelectric properties of filled skutterudite antimonides, we present resonant ultrasound, specific heat, and inelastic neutron scattering results that establish the existence of two low-energy vibrational modes in the filled skutterudite $\mathrm{LaFe}_{3} \mathrm{CoSb}_{12}$. It is likely that at least one of these modes represents the localized, incoherent vibrations of the La ion in an oversized atomic "cage." These results support the usefulness of weakly bound, "rattling" ions for the improvement of thermoelectric performance.

\section{INTRODUCTION}

One of the most promising new ideas in the field of thermoelectrics is the "electroncrystal, phonon-glass" (ECPG) concept originally proposed by Slack [1]. In this picture a loosely bound atom with a large thermal parameter scatters phonons much more strongly than electrons, thus permitting a "glasslike" thermal conductivity to coexist with the high electron mobilities found in crystals. Very recently, Sales et al. [2] reported a high thermoelectric figure of merit (ZT) in $\mathrm{LaFe}_{3} \mathrm{CoSb}_{12}$, which was primarily due to a drastic reduction in the lattice component of the thermal conductivity of this material compared to its unfilled analogue. Although it was hypothesized in Ref. 2 that the reason for the large ZT in filled skutterudites was the incoherent "rattling" of the rare earth ions, no evidence for a low frequency local mode existed at that time. Here we present resonant ultrasound, specific heat, and inelastic neutron scattering results that support the existence of a local mode in $\mathrm{LaFe}_{3} \mathrm{CoSb}_{12}$ and validate the ECPG approach to new thermoelectric materials. 
Polycrystalline samples were synthesized by sealing the starting materials in silica tubes, heating above the liquidus temperature, quenching, annealing, and hot-pressing. All samples were more than $90 \%$ dense, and single phase by powder $x$-ray diffraction. Single crystals were grown using a modified Bridgman method and an Sb-rich melt.

Resonant Ultrasound Spectroscopy (RUS) is a dynamic modulus measurement technique developed by Migliori et al. and reviewed at length in Ref. 3. The technique employs tiny $\mathrm{LiNbO}_{3}$ transducers and a sensitive heterodyne receiver to measure the free-body resonances of a small $\left(2 \times 2.5 \times 3 \mathrm{~mm}^{3}\right)$ sample shaped in the form of a rectangular parallelepiped. Once the freebody resonances are known, non-linear optimization methods are employed to extract the elastic constants.

The neutron experiments were conducted at ORNL's High Flux Isotope Reactor. The single-crystal refinements were performed using a 4-circle neutron diffractometer. The inelastic neutron experiments were performed on a triple axis spectrometer using both pyrolytic graphite and Be energy analyzers.

Standard methods were used for the specific heat and resistivity measurements.

\section{RESULTS AND DISCUSSION}

A brief review of some important crystal-chemical and transport properties of filled skutterudites will be given first. A diagram of the cubic filled skutterudite crystal structure (space group $\operatorname{Im} \overline{3}, 34$ atoms per unit cell) appears in Figure 1. There are two interesting aspects to this structure. First, the rare earth ion sits in an oversized atomic "cage." A typical ionic radius for a rare earth is about $1.4 \AA$, whereas the radius of the "hole" in the skutterudite structure is about $2.0 \AA$. This suggests that the rare earth ion is weakly bound and may have a large thermal parameter. That this is in fact the case may be seen in Figure 2, which illustrates the large difference in thermal parameters between the rare earth ion and the other ions in $\mathrm{LaFe}_{3} \mathrm{CoSb}_{12}$. The second interesting feature in the skutterudite structure is the presence of fourmembered $\mathrm{Sb}$ rings. These rings suggest that the octet principle may in fact be fulfilled in skutterudites if the $\mathrm{Sb}-\mathrm{Sb}$ bonds are properly taken into account. In fact, if we apply a slightly modified form of the octet rule known as the generalized 8-N rule [4], we find that the valence electron concentration per anion (VEC) for skutterudites is 6 . For example, in $\mathrm{CoSb}_{3}$ each Co contributes 9 electrons and each Sb contributes 3 electrons; this gives 18 valence electrons per formula unit, and a VEC of 6 . This implies that skutterudites can be viewed as simple valence compounds if the two Sb-Sb bonds per Sb are taken into account. 'Therefore, the generalized 8-N rule predicts that any skutterudite with a $\mathrm{VEC}=6$ should be a semiconductor, as is $\mathrm{CoSb}_{3}$. In 


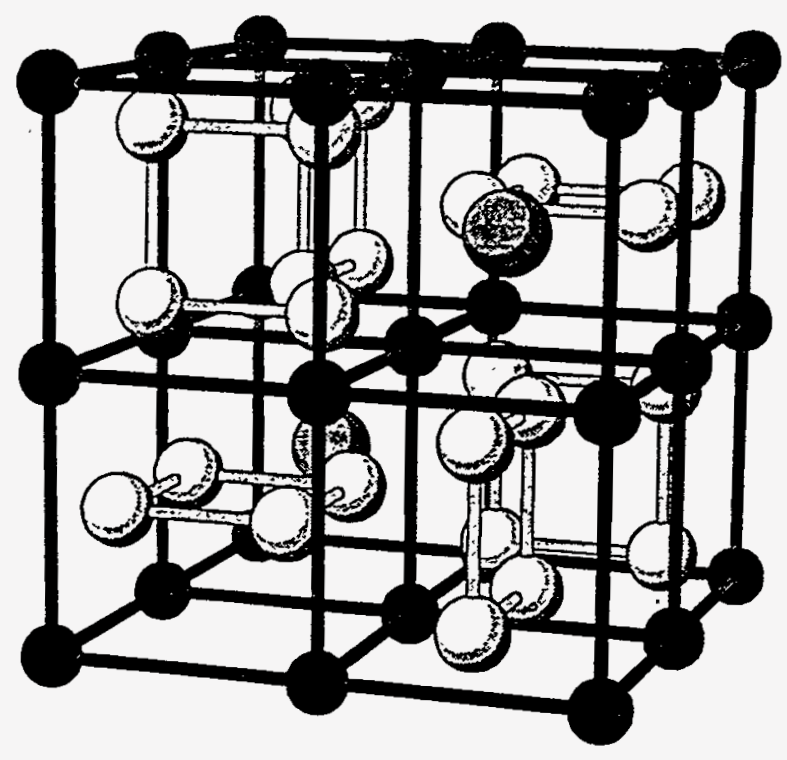

Figure 1. Diagram of the filled skutterudite structure. For $\mathrm{LaFe}_{3} \mathrm{CoSb}_{12}$ the two large balls represent $\mathrm{La}$ ions, the small dark gray balls represent $\mathrm{Fe}$ or $\mathrm{Co}$ ions, and the light gray balls represent $\mathrm{Sb}$ ions. Note the four-membered $\mathrm{Sb}$ rings, and the large "cage" in which the La resides.

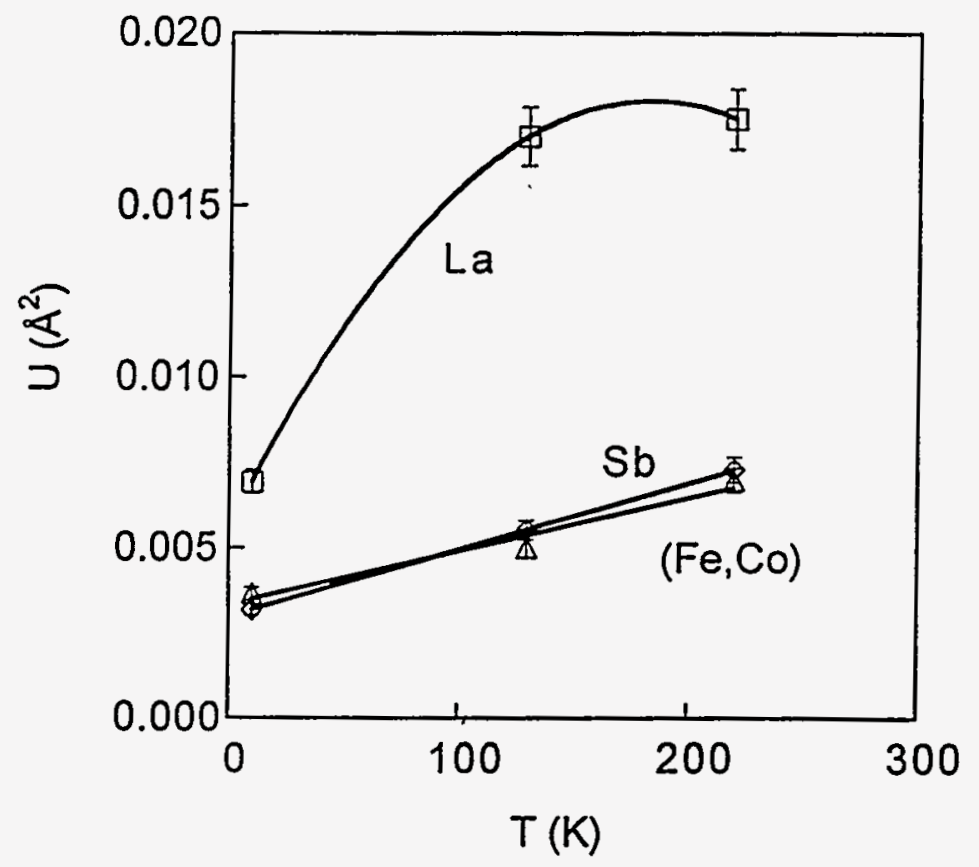

Figure 2. Refined atomic displacement parameters obtained on a single crystal of $\mathrm{La}_{0.75} \mathrm{Fe}_{3} \mathrm{CoSb}_{12}$ using a 4-circle neutron diffractometer. The crystal was approximately $2 \mathrm{~mm}$ on a side. The large value of the atomic displacement parameter for the La ion is consistent with a large amplitude vibratory motion, although static disorder can also lead to a large value of $U$. 


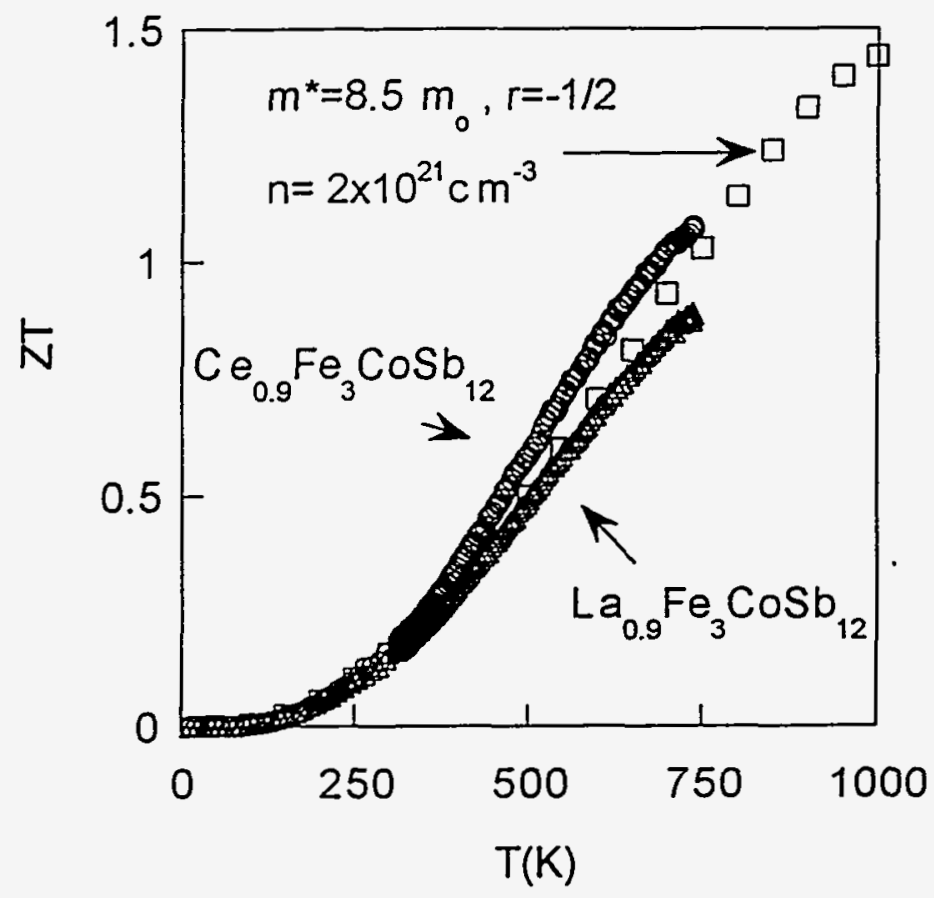

Figure 3. ZT vs. temperature for two filled skutterudite antimonides and a model calculation assuming transport occurs in a single parabolic band. The calculation was performed assuming the relaxation-time approximation, with the energy dependence of the relaxation time given by $\tau=\tau_{0} E^{r}$. For acoustic phonon scattering, $r=-1 / 2$.

semiconducting $\mathrm{LaFe}_{3} \mathrm{CoSb}_{12}$, for example, the $\mathrm{VEC}=6$ and the rule is obeyed. Although these simple electron counting arguments are no substitute for detailed electronic structure calculations [5], they are often useful guides to the synthesis of new materials.

In Figure 3 we plot $Z T$ vs. temperature for two filled skutterudite antimonides. We also plot a model calculation for ZT using generalized Fermi-Dirac statistics and assuming that transport occurs in a single parabolic band. At high temperatures the achievable thermopowers and resistivities of the filled and unfilled materials are not dramatically different, but as we illustrate in Figs. 4 and 5 the lattice component of the thermal conductivity of the filled compounds is an order of magnitude smaller than that of the unfilled at room temperature: Also in Figure 5 we plot the minimum thermal conductivity as calculated by the prescription of Cahill, Watson, and Pohl [6] using sound velocities obtained by RUS. To reach minimum thermal conductivity, heat transport in a material should occur as a random walk of thermal energy between neighboring atoms vibrating with random phases. This implies a mean free path on the order of an interatomic spacing, and is nearly impossible to achieve in real materials. Glasses, for example, have a mean free path of about $10 \AA$. We can estimate the mean free path in $\mathrm{La}_{0.75} \mathrm{Fe}_{3} \mathrm{CoSb}_{12}$ as follows. If we write $\kappa=1 / 3 \mathrm{C}_{v}<v>\Lambda$, where $\mathrm{C}_{v}$ is the heat capacity per unit 


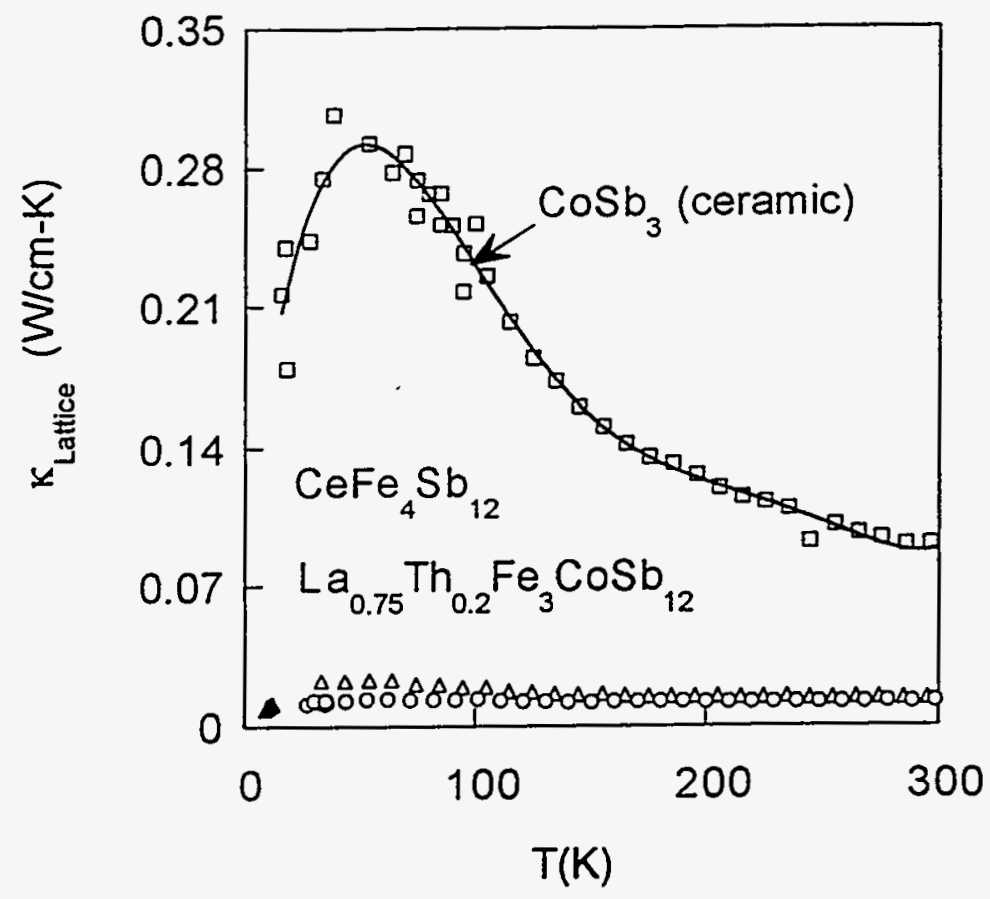

Figure 4. Lattice component of thermal conductivity of $\mathrm{CoSb}_{3}$ and two filled skutterudites vs. temperature. The reduction in thermal conductivity achieved by filling the rare-earth site is truly remarkable.

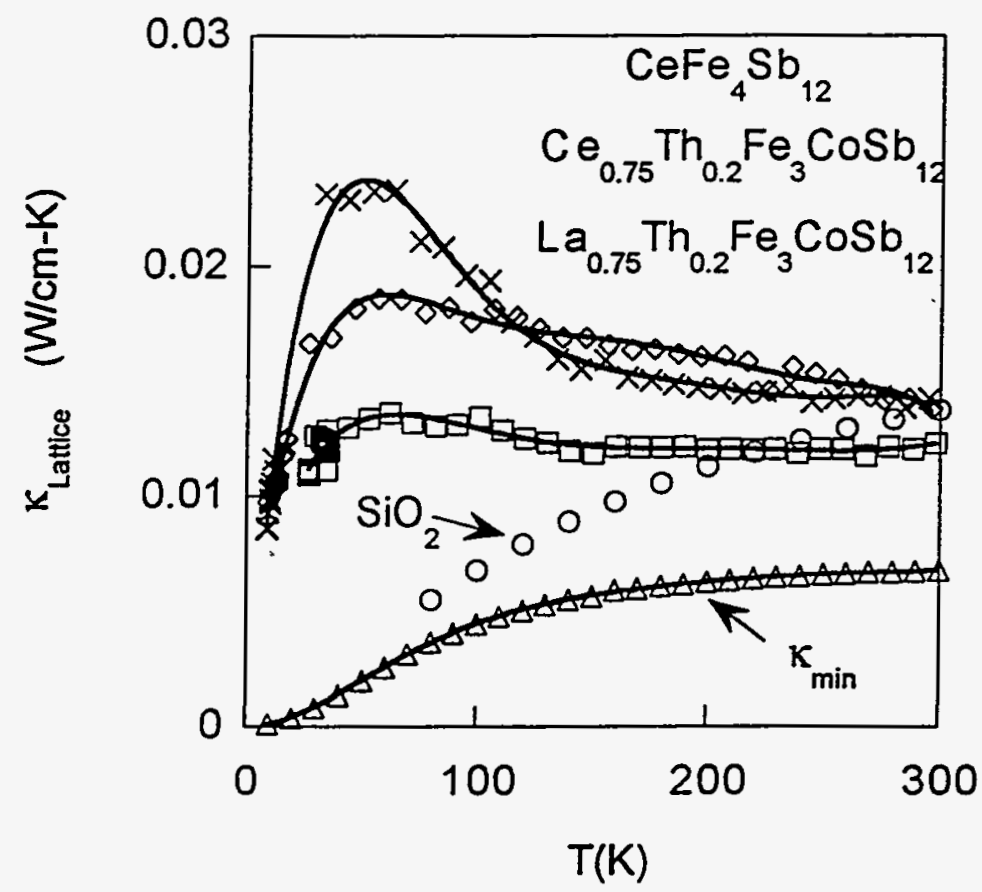

Figure 5. Lattice component of the thermal conductivity of three filled skutterudites and vitreous silica vs. temperature. Also plotted is the minimum thermal conductivity for a filled skutterudite antimonide calculated using Eq. 17 of Ref. 6. 
volume, $\langle v\rangle=$ is the average sound velocity, and $\Lambda$ the phonon mean free path, we find that for $\mathrm{LaFe}_{3} \mathrm{CoSb}_{12}$ we have $\Lambda=8.2 \AA$. This is comparable to the $7.9 \AA$ distance between the rare earth ions, and is consistent with the notion that phonons are scattered by incoherently vibrating La ions.

In order to better characterize the lattice dynamics of filled skutterudites, RUS measurements were performed on both $\mathrm{CoSb}_{3}$ and $\mathrm{La}_{0.75} \mathrm{Fe}_{3} \mathrm{CoSb}_{12}$ from 5 to $300 \mathrm{~K}$. The results appear in Figure 6 and in Table 1. The solid line through the $\mathrm{CoSb}_{3}$ data is a model calculation using the function $c(T)=c_{0}-s /\left(e^{v T}-1\right)$; this function, which has some theoretical justification, was shown by Varshni [7] to describe the temperature dependence of the elastic constants of many simple substances and in effect defines "normal" elastic behavior in much the same way that the Debye model defines "normal" specific heat behavior. It is immediately apparent from Figure 6 that although $\mathrm{CoSb}_{3}$ is well-defined by the Varshni function, the elastic response of $\mathrm{La}_{0.75} \mathrm{Fe}_{3} \mathrm{CoSb}_{12}$ is quite unusual. The large change in $\mathrm{c}_{44}$ at low temperatures is immediately suggestive of a low energy mode that couples strongly to acoustic phonons, and the two welldefined peaks in the ultrasonic absorption of $\mathrm{La}_{0.75} \mathrm{Fe}_{3} \mathrm{CoSb}_{12}$ suggest that two low-energy modes may be present. Although a complete understanding of the elastic response of $\mathrm{La}_{0.75} \mathrm{Fe}_{3} \mathrm{CoSb}_{12}$ is not yet available, we can begin to understand the data by considering the elastic response of a two-level system (TLS). First, we recall that the elastic constants are given by the second derivative of the free energy with respect to strain, $c=\partial^{2} F / \partial \varepsilon^{2}$. The Helmholtz free energy of a TLS is given by $F=-k_{B} T \ln \left(1+e^{-\Delta T}\right)$, where $\Delta$ is the level spacing. If we assume that strain couples to the system as $\Delta=\Delta_{0}+d \varepsilon$, where $d$ is a coupling constant, it is a simple matter to calculate the two-level contribution to the elastic response. As illustrated in Figure 7, we find that we can model the data reasonably well by including 2 TLS's with level spacings of 50 and $200 \mathrm{~K}$.

To further characterize the unusual thermodynamics of filled skutterudites, specific heat measurements were performed on a sample of $\mathrm{La}_{0.9} \mathrm{Fe}_{3} \mathrm{CoSb}_{12}$ from $2 \mathrm{~K}$ to $45 \mathrm{~K}$. The results appear in Figure 8. In addition to the standard $C_{p}=\gamma \mathrm{T}+\beta \mathrm{T}^{3}$, two Einstein oscillators of the form $C_{\text {Einstein }}=\left(\theta_{E} / T\right)^{2} e^{(\theta / T)} /\left(e^{(\theta / T)}-1\right)^{2}$ were required in order to successfully model the data. The Einstein temperatures of the two oscillators were $\theta_{\mathrm{E} 1}=70 \mathrm{~K}$, and $\theta_{\mathrm{E} 2}=157 \mathrm{~K}$. It is tempting to ascribe the lower energy oscillator to the "rattling" of the La ions because the magnitude of the $70 \mathrm{~K}$ oscillator contribution is about what we expect. At high temperature we expect each mole of Einstein oscillators to contribute $3 R=24.94 \mathrm{~J} / \mathrm{K}$ to the heat capacity. Since only about $1 / 17$ of the atoms are "rattlers," we expect a contribution of about $(1 / 17)^{*} 3 \mathrm{R}=1.47$ $\mathrm{J} / \mathrm{mol}-\mathrm{K}$. This is very close to the fitted value of $1.21 \mathrm{~J} / \mathrm{mol}-\mathrm{K}$.

Localized lattice vibrations such as we have been discussing are expected to produce narrow peaks in the phonon density of states (DOS) [8]. In Figures 9 and 10 we present measurements of the phonon DOS using inelastic neutron scattering. These measurements were 


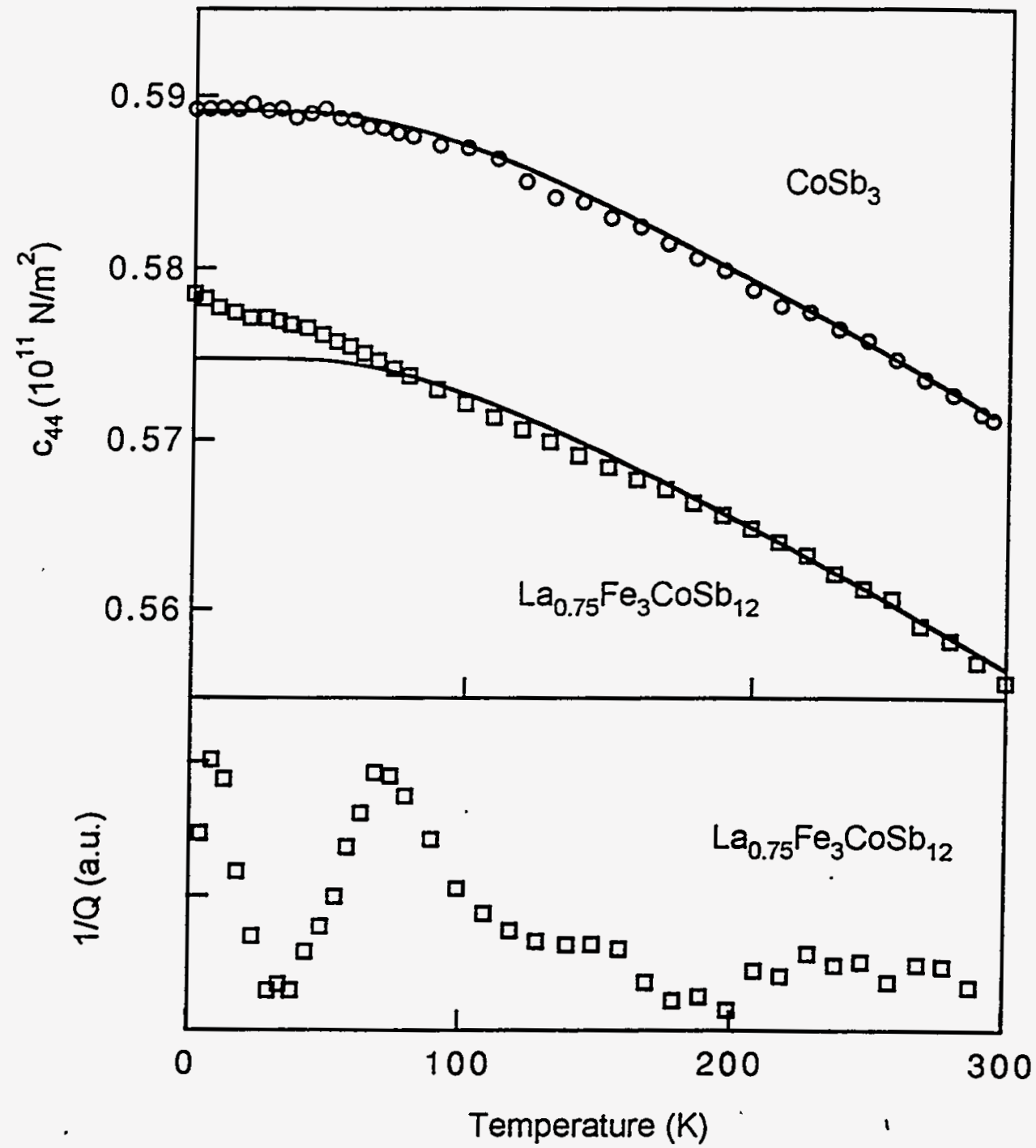

Figure 6. Upper panel. Shear modulus $c_{44}$ vs. temperature for $\mathrm{CoSb}_{3}$ and $\mathrm{La}_{0.75} \mathrm{Fe}_{3} \mathrm{CoSb}_{12}$. The solid line through the $\mathrm{CoSb}_{3}$ data is a fit to the function $c(T)=c_{0}-s /\left(e^{\sqrt{T}}-1\right)$. The fitting parameters used were $c_{0}=0.589 \times 10^{11} \mathrm{~N} / \mathrm{m}^{2}$, $s=0.0275 \times 10^{11} \mathrm{~N} / \mathrm{m}^{2}$, and $t=265 \mathrm{~K}$. The solid line through the $\mathrm{La}_{0.75} \mathrm{Fe}_{3} \mathrm{CoSb}_{12}$ data is the fit to $\mathrm{CoSb}_{3}$ displaced downwards by $0.0144 \times 10^{11}$ $\mathrm{N} / \mathrm{m}^{2}$. Lower panel. $1 / \mathrm{Q}$ vs. temperature for a sample of $\mathrm{La}_{0.75} \mathrm{Fe}_{3} \mathrm{CoSb}_{12}$. Q is the quality factor for a free-body resonance of the sample, and $1 / Q$ is proportional to the ultrasonic absorption. 
Table I. Density, elastic constants, bulk modulus, longitudinal and transverse sound velocity, and Debye temperature for $\mathrm{CoSb}_{3}$ and $\mathrm{La}_{0.75} \mathrm{Fe}_{3} \mathrm{CoSb}_{12}$. These values were derived from RUS measurements.

\begin{tabular}{lcc}
\hline & $\mathrm{CoSb}_{3}$ & $\mathrm{La}_{0.75} \mathrm{Fe}_{3} \mathrm{CoSb}_{12}$ \\
\hline$\rho_{\text {theor. }}\left(\mathrm{g} / \mathrm{cm}^{3}\right)$ & 7.64 & 7.78 \\
$\rho_{\text {exp. }}\left(\mathrm{g} / \mathrm{cm}^{3}\right)$ & 7.29 & 7.64 \\
$c_{11}\left(10^{11} \mathrm{~N} / \mathrm{m}^{2}\right)$ & 1.58 & 1.57 \\
$c_{44}\left(10^{11} \mathrm{~N} / \mathrm{m}^{2}\right)$ & 0.57 & 0.55 \\
$\mathrm{~B}\left(10^{11} \mathrm{~N} / \mathrm{m}^{2}\right)$ & 0.82 & 0.84 \\
$v_{1}(\mathrm{~m} / \mathrm{s})$ & 4650 & 4530 \\
$v_{t}(\mathrm{~m} / \mathrm{s})$ & 2800 & 2680 \\
$\theta_{D}(\mathrm{~K})$ & 319 & 310 \\
\hline
\end{tabular}

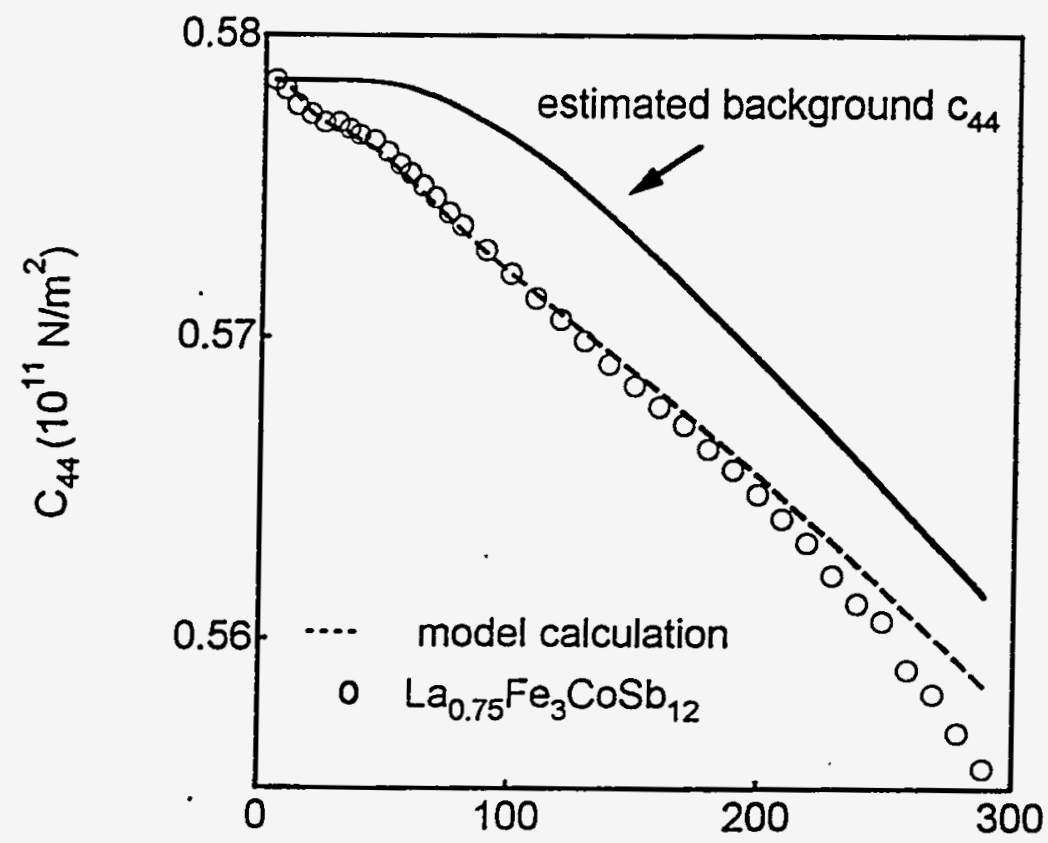

Temperature (K)

Figure 7. Open circles: shear modulus $c_{44}$ vs. temperature for a sample of $\mathrm{La}_{0.75} \mathrm{Fe}_{3} \mathrm{CoSb}_{12}$. Solid line: estimated background $\mathrm{c}_{44}$ obtained from the function $c(T)=c_{0}-s /\left(e^{v T}-1\right)$ taking $s$ and $t$ from the fit to $\mathrm{CoSb}_{3}$ (see Figure 6) and choosing $c_{0}=0.5785 \times 10^{11} \mathrm{~N} / \mathrm{m}^{2}$ to agree with the data at low temperature. Dotted line: two-level system (TLS) model calculation obtained by taking $\mathrm{c}_{44}($ model $)=\mathrm{c}_{44}($ background $)+\mathrm{c}_{44}($ TLS $1, \Delta=50 \mathrm{~K})+\mathrm{c}_{44}(\mathrm{TLS} 2, \Delta=200 \mathrm{~K})$. 


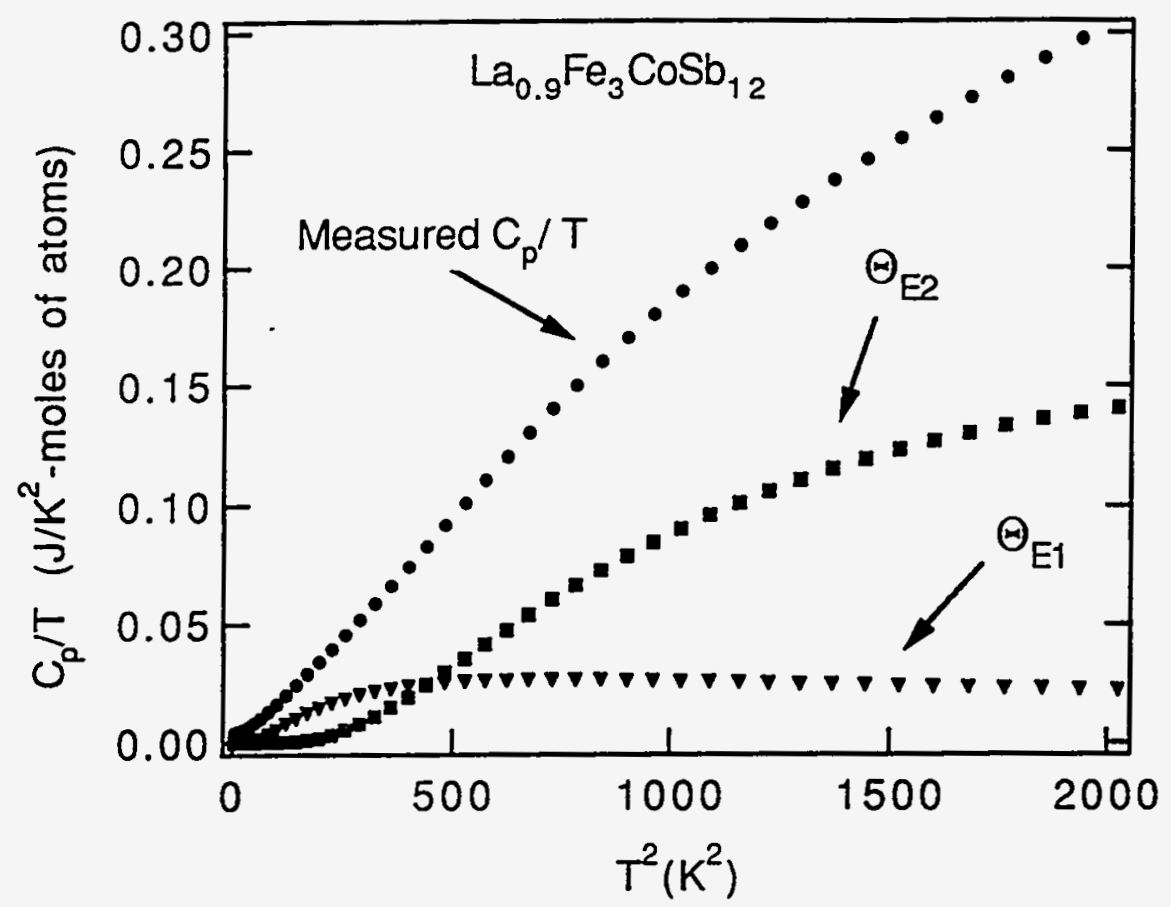

Figure 8. Specific heat $C_{p}$ divided by temperature vs. temperature squared for $\mathrm{La}_{0.9} \mathrm{Fe}_{3} \mathrm{CoSb}_{12}$. The contributions of the two fitted Einstein oscillators are also shown. A fit to the equation $C_{p}(\mathrm{~J} / \mathrm{K}$-mole-atoms $)=\gamma T+\beta T^{3}+A$ $C_{E 1}(T)+B C_{E 2}(T)$ yielded the following values for the fitting parameters: $\gamma=$

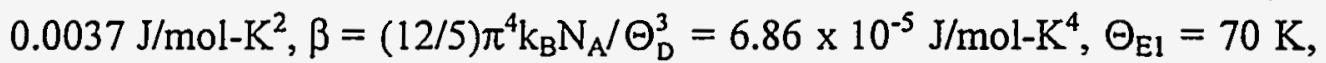
$\mathrm{A}=1.21 \mathrm{~J} / \mathrm{mol}-\mathrm{K}, \Theta_{E 2}=157 \mathrm{~K}, \mathrm{~B}=16.00 \mathrm{~J} / \mathrm{mol}-\mathrm{K}$. The Debye temperature $\Theta_{D}$ obtained from this fit is $\Theta_{D}=302 \mathrm{~K}$. This is in excellent agreement with the Debye temperature of $309 \mathrm{~K}$ obtained using RUS (see Table 1).

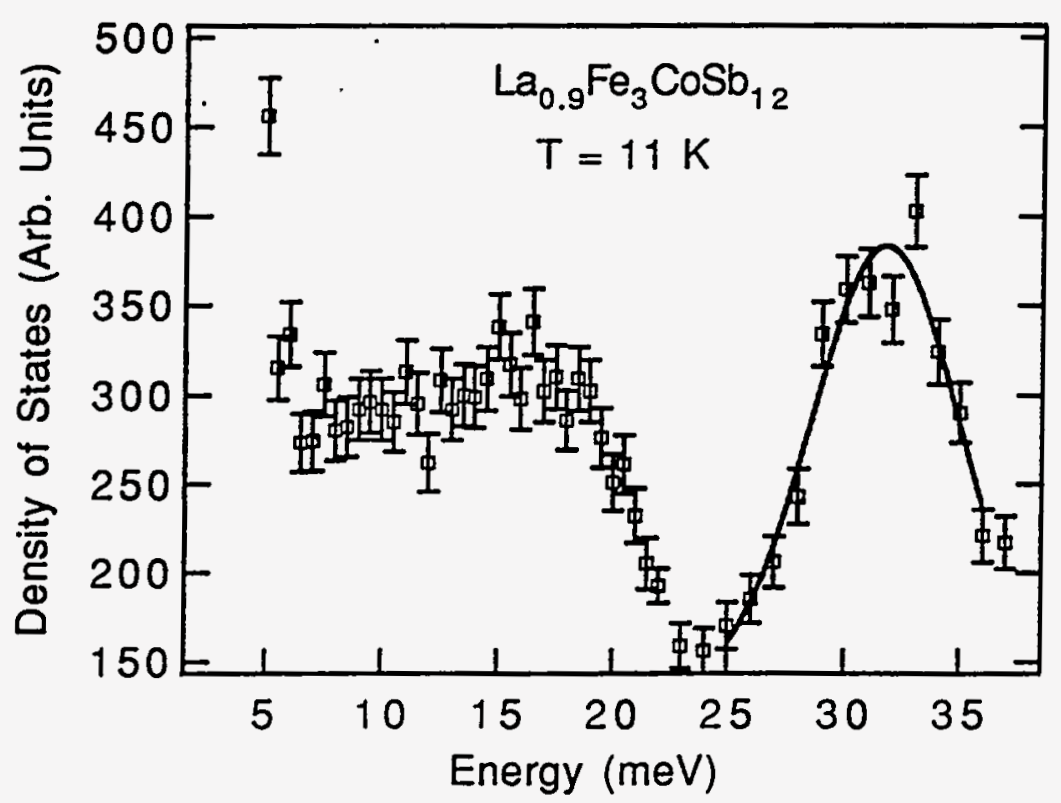

Figure 9. Phonon density of states for $\mathrm{La}_{0.9} \mathrm{Fe}_{3} \mathrm{CoSb}_{12}$ obtained by inelastic neutron scattering using a pyrolytic graphite energy analyzer. The solid line represents a Gaussian fit to the peak with a center energy of $31.7 \mathrm{meV}$. 


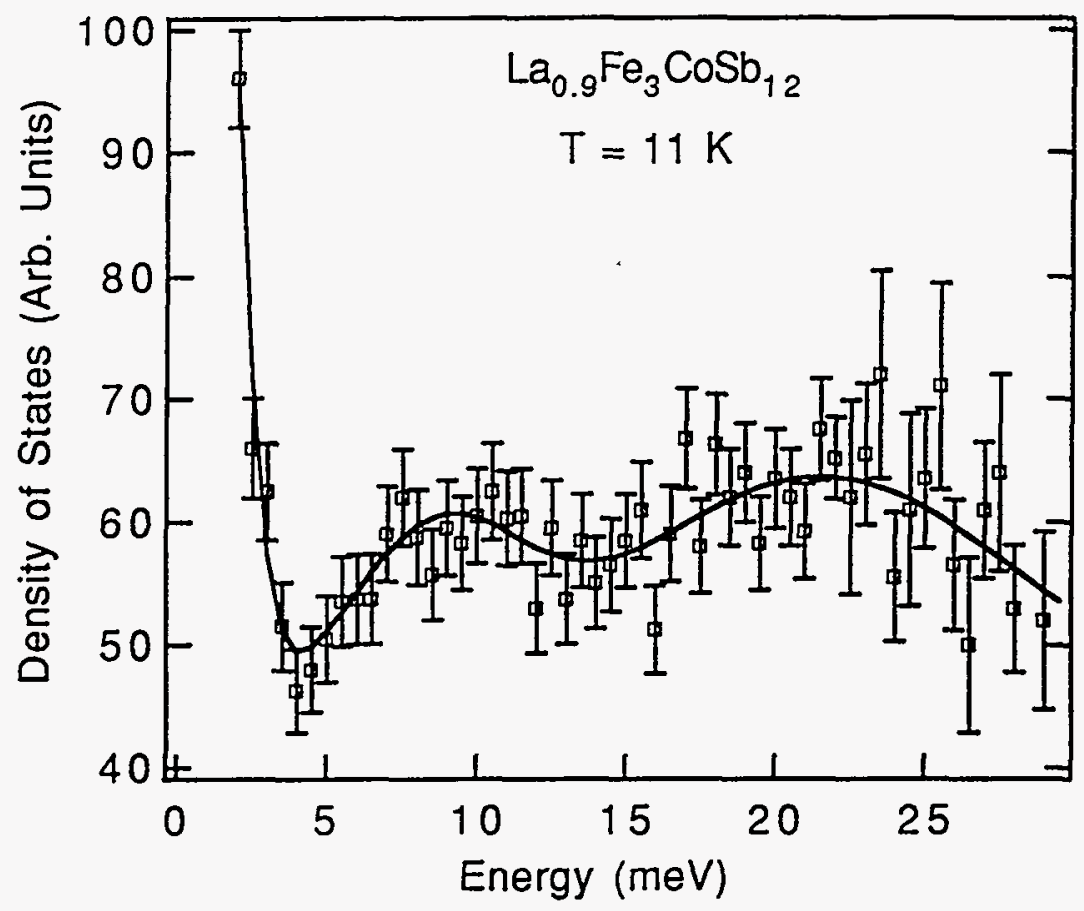

Figure 10. Phonon density of states for $\mathrm{La}_{0.9} \mathrm{Fe}_{3} \mathrm{CoSb}_{12}$ obtained using inelastic neutron scattering and a high resolution $\mathrm{Be}$ analyzer.

performed at $11 \mathrm{~K}$ on a $30 \mathrm{~g}$ polycrystalline sample of $\mathrm{La}_{0.9} \mathrm{Fe}_{3} \mathrm{CoSb}_{12}$. Using a high resolution $\mathrm{Be}$ analyzer and 4 days of counting time, two low energy peaks were visible, one at $100 \mathrm{~K}$ and one at $240 \mathrm{~K}$. Although the energies of these peaks are close to the vibrational energies observed in the ultrasound and specific heat experiments, it is important to note that a calculation of the phonon DOS of $\mathrm{CoSb}_{3}$ by Feldman and Singh [9] predicts a double peak structure somewhat similar to the one observed in $\mathrm{La}_{0.9} \mathrm{Fe}_{3} \mathrm{CoSb}_{12}$. Given that RUS has shown that the lattice dynamics of $\mathrm{CoSb}_{3}$ and $\mathrm{La}_{0.9} \mathrm{Fe}_{3} \mathrm{CoSb}_{12}$ are very different, it is unclear how much weight to attach to these calculations in the evaluation of data on the filled materials. Measurements of the phonon DOS of $\mathrm{CoSb}_{3}$ are presently underway, and these should help us determine the source of the peaks in the phonon DOS of $\mathrm{La}_{0.9} \mathrm{Fe}_{3} \mathrm{CoSb}_{12}$.

In conclusion, the filled skutterudite antimonides appear to represent excellent examples of electron-crystal, phonon-glass materials in which the incoherent "rattling" of loosely bound ions greatly reduces the lattice component of the thermal conductivity while only modestly degrading the electronic transport properties. Resonant ultrasound, specific heat, and inelastic neutron measurements have indicated the existence of two low-energy vibrational modes in $\mathrm{La}_{0.9} \mathrm{Fe}_{3} \mathrm{CoSb}_{12}$ that do not appear to be present in $\mathrm{CoSb}_{3}$. It is likely that one, or both, of these modes are ássociated with the vibrations of the La ion in a shallow potential well. 
Oak Ridge National Laboratory is managed by the Lockheed Martin Energy Research Corporation for the U.S. Department of Energy under contract No. DE-AC05-960R22464. Work at Los Alamos was performed under the auspices of the U.S. Department of Energy.

\section{REFERENCES}

[1] G. A. Slack in Thermoelectric Handbook, CRC Press, Boca Raton, FL, 1995.

[2] B. C. Sales, D. Mandrus, and R. K. Williams, Science 272, p. 1325 (1996).

[3] A. Migliori, J. L. Sarrao, W. M. Visscher, T. M. Bell, M. Lei, Z. Fisk, and R. G. Leisure, Physica B 183, p. 1 (1993).

[4] For a lucid exposition see U. Müller, Inorganic Structural Chemistry, John Wiley, New York, 1993, pp. 116-126.

[5] It is in fact surprising that the generalized 8-N rule appears to work in skutterudites because calculations by D. J. Singh and W. E. Pickett, Phys. Rev. B 50, p. 11235 (1994) indicate little static charge transfer between the pnicogen and transition metal.

[6] D. G. Cahill, S. K. Watson, and R. O. Pohl, Phys. Rev. B 46, p. 6131 (1992).

[7] Y. P. Varshni, Phys. Rev. B 2, p. 3952 (1970).

[8] A. D. Caplain and L. K. Nicholson, J. Phys. F 8, p. 51 (1978).

[9] J. L. Feldman and D. J. Singh, Phys. Rev. B 53, p. 6273 (1996). 